\title{
FGF8 initiates inner ear induction in chick and mouse
}

\author{
Raj K. Ladher, ${ }^{1,2,6,7}$ Tracy J. Wright, ${ }^{4,6}$ Anne M. Moon, ${ }^{2,5}$ Suzanne L. Mansour, ${ }^{2,4}$ and \\ Gary C. Schoenwolf ${ }^{2,3}$ \\ ${ }^{1}$ Sensory Development, Riken Center for Developmental Biology, Chuo-ku, Kobe 650-0047, Japan; ${ }^{2}$ Department \\ of Neurobiology and Anatomy and ${ }^{3}$ Children's Health Research Center, University of Utah School of Medicine, \\ Salt Lake City, Utah 84132-3401, USA; ${ }^{4}$ Department of Human Genetics and ${ }^{5}$ Program in Human Molecular Biology \\ and Genetics, University of Utah, Salt Lake City, Utah 84112-5330, USA
}

\begin{abstract}
In both chick and mouse, the otic placode, the rudiment of the inner ear, is induced by at least two signals, one from the cephalic paraxial mesoderm and the other from the neural ectoderm. In chick, the mesodermal signal, FGF19, induces neural ectoderm to express additional signals, including WNT8c and FGF3, resulting in induction of the otic placode. In mouse, mesodermal Fgf10 acting redundantly with neural Fgf3 is required for induction of the placode. To determine how the mesodermal inducers of the otic placode are localized, we took advantage of the unique strengths of the two model organisms. We show that endoderm is necessary for otic induction in the chick and that Fgf8, expressed in the chick endoderm subjacent to Fgf19, is both sufficient and necessary for the expression of Fgf19 in the mesoderm. In the mouse, Fgf8 is also expressed in endoderm as well as in other germ layers in the periotic placode region. We show that otic induction fails in embryos null for $\mathrm{Fgf3}$ and hypomorphic for $\mathrm{Fgf8}$ and expression of mesodermal $\mathrm{Fg} f 10$ is reduced. Thus, Fgf8 plays a critical upstream role in an FGF signaling cascade required for otic induction in chick and mouse.
\end{abstract}

[Keywords: FGF8; FGF3; FGF10; FGF19; otic; endoderm]

Received October 19, 2004; revised version accepted January 14, 2005.

The inner ear is derived from the otic placode, a thickened disc of nonneural ectoderm that appears early in development and lies adjacent to the caudal hindbrain. In both chick and mouse embryos, the placode is morphologically visible lateral to the fifth and sixth rhombomeres (r) at approximately the eight-somite stage (Anniko and Wikstrom 1984; Alvarez and Navascues 1990), but is specified earlier. Namely, in explants of chick head ectoderm at the five-somite stage (stage $8+$ ), the otic ectoderm is already specified with respect to $\mathrm{Pax} 2$ expression (Groves and Bronner-Fraser 2000). Although similar culture experiments have not been performed in other species, it is clear from these experiments that induction of the otic placode occurs soon after neurulation is initiated.

A rich history of embryological manipulations has led to a well-understood pattern of tissue interactions during otic placode induction (for reviews, see Jacobson 1966; Baker and Bronner-Fraser 2001; Riley and Phillips 2003). These studies, performed in either amphibian or avian embryos, suggest a two-signal model for otic induction in which otic inducers located in the head mesoderm and

\footnotetext{
${ }^{6}$ These authors contributed equally to the study.

${ }^{7}$ Corresponding author.

E-MAIL raj-ladher@cdb.riken.go.jp; FAX 81-78-306-3322

Article and publication are at http://www.genesdev.org/cgi/doi/10.1101/ gad. 1273605
}

neural ectoderm (caudal hindbrain) are required and act in concert for complete otocyst development.

Numerous molecular candidates have been postulated as either neural or mesodermal inducers of the otic placode. Different species seem to use different molecules. However, most are members of the fibroblast growth factor (FGF) family of secreted signaling proteins, which communicate with nearby cells by activating FGF receptor tyrosine kinases. In the chick, one such member, Fgf19, is localized to the cranial paraxial mesoderm at the otic placode-forming level, where it initiates a network of signaling interactions that results in otic induction. By signaling to the neural ectoderm, FGF19 induces the expression of $W n t 8 c$. Together these molecules synergize to induce the otic placode (Ladher et al. 2000). Overexpression studies (Vendrell et al. 2000) also show a role for Fgf3 in the induction of an otic fate. Fgf3 can induce adjacent ectoderm to form small ectopic otocysts (Vendrell et al. 2000), whereas reducing Fgf3 action results in a hypoplastic vesicle (Represa et al. 1991). However, as these latter experiments were performed after the otic placode had already formed, it is unclear where Fgf3 normally belongs in the signaling hierarchy for placode induction in the chick. Gain-of-function experiments also suggest a role for FGF2 and FGF8 during otic development. Beads soaked in FGF2 induced patches of hypoplastic otocysts (Adamska et al. 2001). The ubiquitous expression of Fgf2 at these stages (Karabagli et al. 
2002) makes the exact role of this factor difficult to determine. In the study of Adamska et al. (2001), FGF8 beads were also tested, and these caused an enlargement of the otic placode and an alteration of the pattern of gene expression within the otocyst, but did not induce ectopic otocysts. As Fgf8 is expressed transiently in the otic cup and otocyst, these findings suggest that Fgf8 might function in later otic patterning.

The murine ortholog of Fgf19 is Fgf15. However, its expression pattern does not obviously suggest a role in otic induction. Moreover, Fgf15 null mutants do not show overt otic abnormalities (Wright et al. 2004). Instead, Fgf10 expressed in the periotic mesoderm, together with Fgf3 expressed in the caudal hindbrain, are required redundantly to induce the mouse otic placode (Wright and Mansour 2003a).

The mechanism of otic induction by FGFs in zebrafish seems to be different. In this species, Fgf3 and $F g f 8$ are required redundantly for otic induction (Phillips et al. 2001; Leger and Brand 2002; Maroon et al. 2002). Both genes are expressed in the involuting germ ring of the gastrula. In contrast to $F g f 8, F g f 3$ expression persists in the prechordal mesoderm and cephalic paraxial mesoderm at $80 \%$ epiboly (Phillips et al. 2001). Both Fgf3 and Fgf8 are then expressed in $\mathrm{r} 4$, near the site at which the otic placode forms. Fgf8 is also expressed in the cardiac mesoderm, which lies anterior to, but in close proximity to, the future otic placode (Reifers et al. 1998, 2000). Thus, there are several sites from which Fgf3 and Fgf8 might influence zebrafish otic induction. In contrast to zebrafish, expression of $F g f 8$ in chick or mouse paraotic hindbrain has not been reported, and, consequently, potential requirements for Fgf8 in otic placode induction have not been addressed previously in these species.

We report here that Fgf8 has an early role in otic induction in both chick and mouse. We show that endoderm is necessary for otic induction in the chick and that Fgf8, expressed in the chick endoderm subjacent to Fgf19, is both sufficient and necessary for the expression of Fgf19 in the mesoderm. We extend these results to the mouse and show that $F g f 8$ is expressed in the prospective otic placode, as well as in the subjacent head mesenchyme and endoderm. Otic induction fails in embryos lacking Fgf3 and having severely reduced levels of Fgf8. Furthermore, the otic phenotypes of embryos with both combinations of three mutant Fgf3 and Fgf8 alleles are very similar to those of embryos with analogous combinations of Fgf3 and Fgf10 null alleles. Finally, we show that $F g f 8$ is required redundantly with Fgf3 for normal expression of the murine mesodermal otic inducer, Fgf10. Thus in both species, Fgf8 plays a critical role in induction of the mesodermal otic inducer.

\section{Results}

\section{Endoderm is required for otic induction}

We have previously described roles for Fgf19-expressing mesoderm and Wnt8c-expressing neural ectoderm in induction of chick otic placode (Ladher et al. 2000). A third tissue, the endoderm, lies in close proximity to these tissues. To assess the role of endoderm in otic induction, we unilaterally ablated the cranial endoderm, including that located beneath the Fgf19-expressing region, and we examined otic development morphologically and with the otic marker Pax2. Endoderm was ablated at stage 5, prior to otic induction (Fig. 1A), resulting in a hypoplastic or absent otic placode on the operated side $(n=23 / 40$; Fig. 1B,C). Typically, endoderm removal did not cause complete loss of the otocyst or Pax2 labeling, presumably because the endoderm rapidly regenerated, as revealed in serial sections (data not shown). The high frequency of otocyst loss following endoderm ablation strongly indicates that endoderm is necessary for inner ear induction.

\section{Endoderm induces chick mesodermal Fgf19 expression}

The endoderm is not in contact with the prospective or definitive otic placode, suggesting that its role in otic induction is indirect. To ask whether endoderm induced expression of the mesodermal inducer, Fgf19, we used tissue explants. Anterior mesoderm was isolated from stage 5 embryos and stripped of adhering ectoderm and endoderm (Fig. 2A, left panel). Fgf19 was not expressed in these isolates $(n=0 / 12$; Fig. $2 \mathrm{~B})$, even after $8 \mathrm{~h}$ of culture, a stage at which unstripped mesoderm expressed $F g f 19$ $(n=3 / 4$; data not shown). We next used this assay to test the ability of quail endoderm to induce Fgf19. To ensure that only mesodermal and not endodermal Fgf19 expression was detected, we prepared quail endoderm-chick mesoderm chimeric explants and used a probe directed against the 3' UTR of chick Fgf19. For ease of endoderm dissection we used stage 4 quail donors and isolated anterior and posterior endoderm (Fig. 2A, right panel). Only

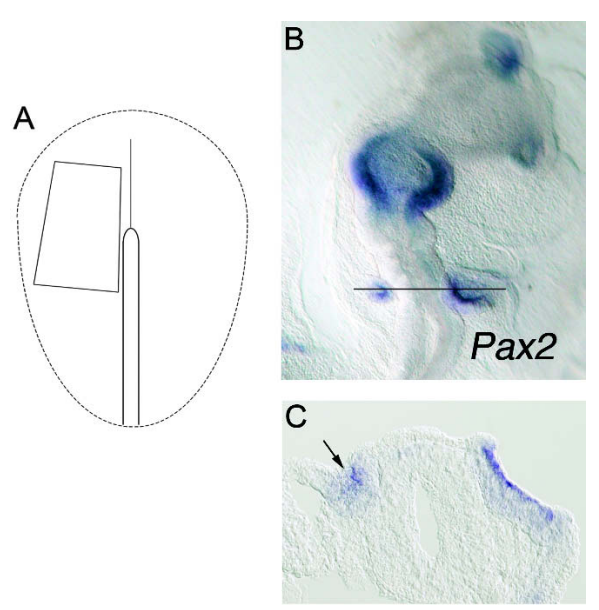

Figure 1. Endoderm ablation blocks otic development. The endoderm was removed unilaterally from chick embryos, which were cultured for 24-36 $\mathrm{h}$ and then assayed for the otic marker, Pax2. (A) Diagram illustrating endoderm removal (box). (B) Dorsal view showing a smaller otic domain of $P a x 2$ on the operated (left) side. Black line indicates axial level of section in $C .(C)$ Section showing the smaller left otic placode (arrow). 


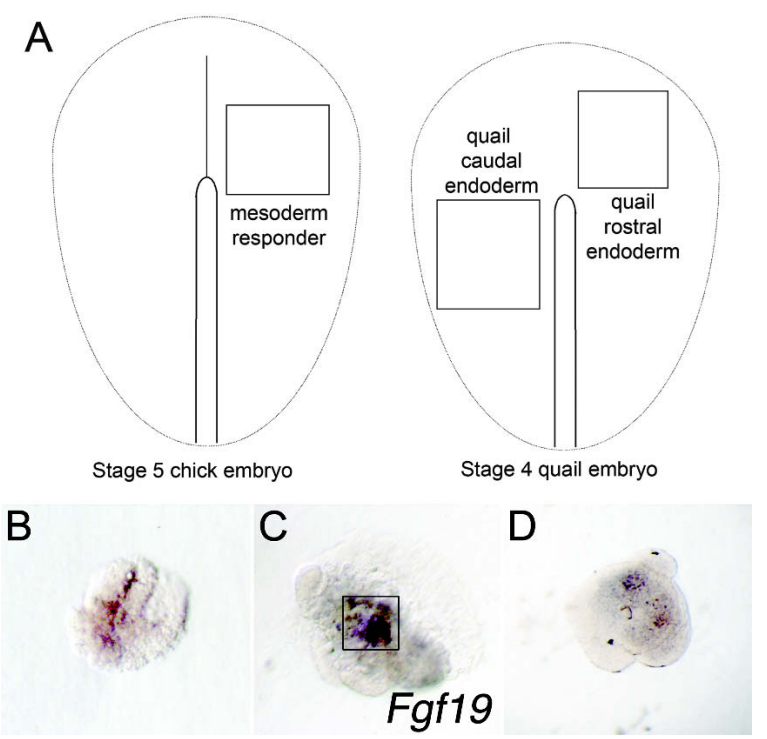

Figure 2. Mesodermal Fgf19 expression requires subjacent endoderm. Explanted stage 5 rostral chick mesoderm was cultured alone or with stage 4 quail endoderm. Explants were fixed when staged-matched whole-embryo controls had reached stage 8and then hybridized with a chick-specific Fgf19 probe. (A) Box in left diagram indicates the mesoderm explanted. Boxes in right diagram indicate the endoderm explanted. $(B)$ Mesodermal explants stripped of ectoderm and endoderm show only background labeling. $(C)$ When recombined with caudal endoderm, mesodermal explants express Fgf19 (boxed). (D) Rostral endoderm fails to induce Fgf19.

the posterior endoderm is fated to underlie the Fgf19expressing mesoderm; the more rostral endoderm is displaced more anteriorly (Lawson and Schoenwolf 2003). Chick mesodermal Fgf19 expression occurred when chick mesoderm was recombined with posterior quail endoderm $(n=6 / 8$; Fig. $2 \mathrm{C})$. In contrast, anterior quail endoderm did not induce Fgf19 expression $(n=0 / 8$; Fig. $2 \mathrm{D})$. Thus, the endoderm underlying the otic-inducing mesoderm is sufficient to induce Fgf19 expression, thereby initiating otic induction.

Expression of Fgf8 is spatially and temporally appropriate for a role in mesodermal Fgf19 induction

We next considered which endodermally expressed signaling molecules might be candidates for the inducer of mesodermal Fgf19. We focused on Fgf8. Beginning at stage $6, F g f 8$ was expressed in the cranial endoderm in two domains (Fig. 3A). The most obvious domain marked the endoderm in a horseshoe-shaped region underlying the developing heart rudiments. The second domain marked a transverse stripe of endoderm, rostral to the primitive streak (Fig. 3A,B). This latter domain was subjacent to the Fgf19-expressing mesoderm (Fig. 3, cf. $\mathrm{A}, \mathrm{B}$ and $\mathrm{C}, \mathrm{D})$. Fgf8 expression in the transverse domain became stronger as the embryo aged, and it changed from a punctate pattern to a uniform and strong expression domain at stage 8 (Fig. 3E). The transverse endodermal domain of Fgf8 expression remained subjacent to the Fgf19-expressing mesoderm during subsequent development (Fig. 3, cf. E and F).

\section{FGF8 induces Fgf19 in chick mesodermal isolates}

To determine whether FGF8 induces mesodermal Fgf19, we cocultured mesodermal explants with heparin beads soaked in FGF4 or FGF8 for 6-8 h. Isolates cultured alone $(n=0 / 15$; Fig. 4A) or with FGF4 ( $n=2 / 16$; Fig. $4 \mathrm{~B})$ did not express Fgf19. In contrast, isolates cultured with FGF8 expressed $F g f 19$ ( $n=16 / 20$; Fig. 4C). These results show that FGF8 is sufficient for induction of Fgf19.

\section{Fgf8 is necessary for initial chick mesodermal Fgf19 expression and otic placode induction}

We next asked whether endodermal Fgf8 is necessary for induction of mesodermal Fgf19 by inhibiting FGF8 expression in vivo. We unilaterally introduced an Fgf8 hairpin RNA-expressing vector together with a Venus tracer plasmid (Fig. 5A). The vector-derived Fgf8 siRNA effectively targeted endogenous F $f 8$ mRNA for destruction $(n=12 / 14$; Fig. 5B). In embryos where Venus was expressed in the presumptive otic-inducing region, Fgf19 transcripts were down-regulated by $6-12 \mathrm{~h}(n=16 / 22$; Fig. 5C-E). When cultured to stage 12, the embryos expressing Fgf8 siRNA had a reduced or absent otic placode

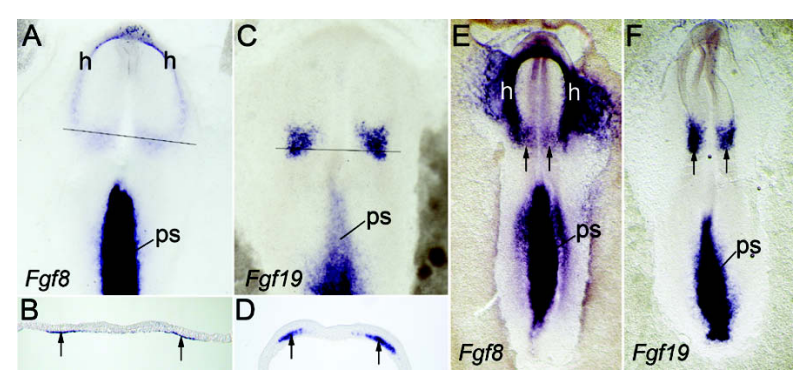

Figure 3. Chick $F g f 8$ is expressed in the endoderm underlying mesodermal Fgf19. Chick embryos processed for in situ hybridization with the indicated probes. Lines in $A$ and $C$ indicate the levels of the sections shown in $B$ and $D$, respectively. (A) Dorsal view of a stage 6 (zero-somite) embryo showing two expression domains of Fgf8 in the cranial endoderm: a horseshoe-shaped area underlying the developing heart rudiments $(\mathrm{h})$ and a transverse domain rostral to the primitive streak (ps). (B) Section showing Fgf8 expression in transverse endodermal domain (arrows). (C) Dorsal view of a stage 6 embryo showing Fgf19 expression in the primitive streak (ps) and in bilateral patches of paraxial mesoderm overlying the Fgf8 transverse-endodermal domain. (D) Section showing bilateral Fgf19 expression in the paraxial mesoderm (arrows). (E) Dorsal view of a stage 8 (threesomite) embryo showing Fgf8 expression in the primitive streak (ps), cranial endodermal domains, and endoderm underlying heart rudiments (h). Note the transverse stripe of endodermal expression (arrows) just rostral to the first pair of somites. $(F)$ Dorsal view of a stage 8 embryo showing Fgf19 expression in the primitive streak (ps) and bilateral paraxial mesodermal patches (arrows) just rostral to the first pair of somites. 
Ladher et al.

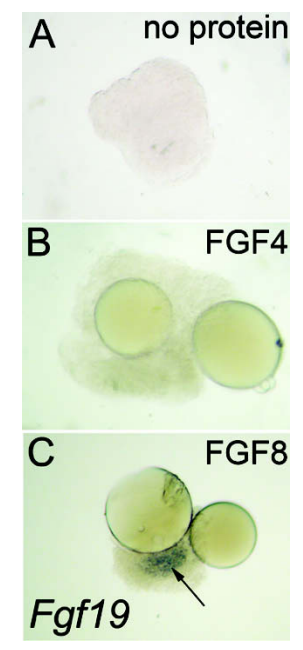

Figure 4. FGF8 is sufficient for Fgf19 expression. Stripped stage 5 chick mesoderm was cultured in isolation or in the presence of either FGF4 or FGF8. (A) Untreated mesodermal explants fail to express $F g f 19$ after $8 \mathrm{~h}$ of culture. $(B)$ Mesodermal explants treated with FGF4 beads also do not express Fgf19. (C) Mesodermal explants treated with FGF8 beads express Fgf19 (arrow).

on the electroporated side, as indicated by a reduction in Pax2 expression and the absence of normal placodal morphology $(n=18 / 25$; Fig. 5F-H). In controls, using either the Venus construct alone or an siRNA vector containing a scrambled $F g f 8$ hairpin construct, $F g f 19$ expression and the otic placode were unaffected (data not shown). Thus Fgf8 is required for Fgf19 expression and otic induction.

\section{Fgf8 mediates otic induction through induction of Fgf19}

To determine whether loss of $F g f 19$ was responsible for the block to otic development, we electroporated the Fgf8 siRNA construct as before, explanted the three-layered presumptive otic/periotic region (Fig. 5I; explant " $\mathrm{a}$ " of Ladher et al. [2000]), cultured it in the presence or absence of FGF19 beads, and assayed for Pax2 expression. An unelectroporated control explant expressed Pax2 as expected (Fig. 5J). In the absence of FGF19, the Fgf8 siRNA-exposed explants failed to express Pax2 (Fig. $5 \mathrm{~K} ; n=4 / 6$ explants negative for Pax2), whereas similar explants treated with FGF19 expressed Pax2 (Fig. 5L; $n=4 / 6$ explants positive for Pax2). Thus, FGF19 rescues Pax2 expression, indicating that Fgf8 acts indirectly on otic induction.

Fgf8 is also expressed in a pattern consistent with a role in early mouse otic development

We show above that $F g f 8$ is both sufficient and necessary for the expression of the chick mesodermal otic inducer, Fgf19. To determine whether this role for Fgf8 was conserved in the mouse we focused attention on the early expression domains relative to the developing otic placode using both in situ hybridization to Fgf 8 mRNA in wild-type embryos and immunohistochemistry to an FGF8/GFP fusion protein expressed from a targeted Fgf8 allele (Macatee et al. 2003). At embryonic day 7.0 (E7.0), Fgf8 transcripts were found in the primitive streak and in mesoderm underlying the lateral neural plate (Fig. 6A,B). By E8.0, just before the embryo developed its first somite, Fgf8 expression in the primitive streak expanded and was also apparent in the splanchnic (heart-forming) mesoderm, which lies lateral and ventral to the preplacodal ectoderm (Fig. 6C,D). By the three-somite stage, all mesenchyme underlying the prospective placode expressed the FGF8/GFP fusion protein (Fig. 6E). At the four-somite stage, Fgf 8 transcripts were found in preplacodal ectoderm, as well as in more ventral ectoderm,

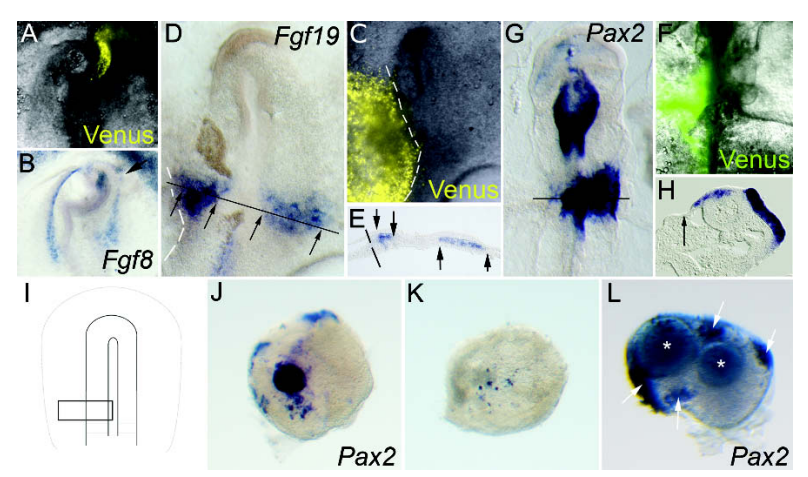

Figure 5. FGF8 is necessary for Fgf19 expression and inner ear induction. Two plasmids, a Histone-2B/Venus fusion construct and pSilencer- $F g f 8$, were coelectroporated on one side of stage 4 chick embryos. Treated embryos were cultured for 4-8 or 24$36 \mathrm{~h}$, observed with fluorescent illumination, and then processed for in situ hybridization with probes as indicated. $(A)$ Venus fluorescence (yellow) indicates the electroporated area. (B) Fgf8 transcripts are down-regulated in the region of Venus expression (arrow). (C-E) At stage 7, 4-8 h after electroporation, embryos treated with the pSilencer-Fgf 8 vector show a lateral reduction in Fgf19 expression. (C) Only embryos that showed appropriately targeted Venus fluorescence (yellow) were analyzed. $(D)$ Whole mount showing a loss of lateral Fgf19 expression on the electroporated (left) side. Arrows in $D$ and $E$ mark the medial-lateral extent of expression on both the right and left sides. Line marks the level of the section in E. $(E)$ Section showing loss of $F g f 19$ expression in the lateral paraxial mesoderm on the electroporated (left) side. $(F-H)$ At stages 12-14 (24-36 h after electroporation), embryos treated with the pSilencer-Fgf8 vector show a loss of $P a x 2$ expression and failure of placodal morphology to form on the electroporated (left) side. $(F)$ Only embryos that showed appropriately targeted Venus fluorescence (yellow) were analyzed. (G) Whole mount showing a loss of Pax2 expression on the electroporated side. Line marks the level of the section in $H$. (H) Section showing loss of Pax2 expression on the electroporated (left) side. In addition, placodal morphology fails to form (arrow), as is evident when compared with the normal Pax2-expressing placode on the right side. (I) Location of three-layered explants (boxed) used in rescue experiments. $(J)$ Control explants express Pax2. (K) Fgf8 siRNA electroporated explants fail to express Pax2. (L) Electroporated explants treated with FGF19 beads (asterisks) express Pax2 (arrows). 


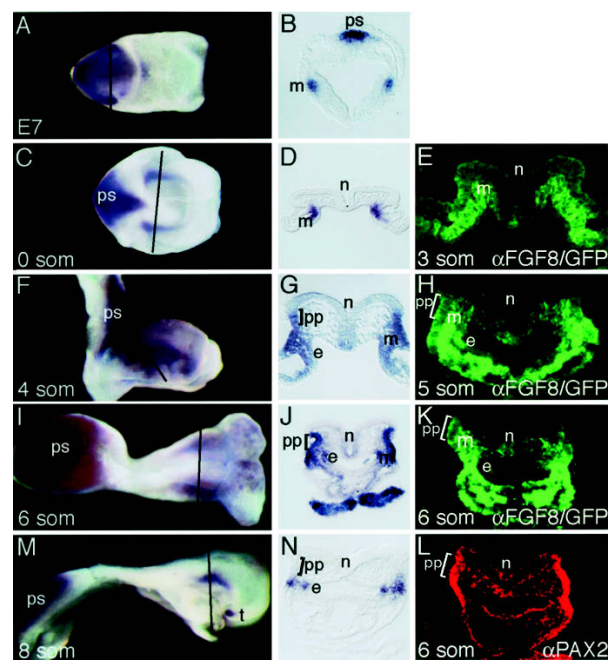

Figure 6. Mouse $F g f 8$ is expressed in tissues relevant to early otic development. $(A, C, F, I, M)$ E7.0-E8.0 mouse embryos hybridized with an $F g f 8$ probe and sectioned in the transverse plane. $(B, D, G, J, N)$ Sections through the otic region (the plane is indicated by a line through each embryo) are shown in the panel to the right of each whole embryo. Whole-mount images are shown in the dorsal $(A, C, I)$ or lateral $(F, M)$ views. Anterior is to the right. $(E, H, K)$ Mouse embryos bearing the Fgf ${ }^{\mathrm{GFPR}}$ allele were sectioned and the FGF8/GFP fusion protein was detected using immunohistochemsitry directed against GFP (green). ( $L)$ PAX2 immunohistochemistry (red) was performed on a section adjacent to that shown in $K .(A, B)$ At E7, Fgf8 is expressed in the heart mesoderm $(\mathrm{m})$ and in the primitive streak (ps). (C,D) Fgf8 expression at zero somites in splanchnic mesoderm $(\mathrm{m})$ and primitive streak (ps). (E) At the three-somite stage, the FGF8/ GFP fusion protein is localized to splanchnic mesoderm. $(F-K)$ At four to six somites, $F g f 8$ transcripts and the FGF8/GFP fusion protein are expressed throughout the surface ectoderm, including the prospective placode (pp), pharyngeal endoderm (e), and the splanchnic as well as more dorsal, paraxial, mesoderm (m) and in the primitive streak (ps). At these stages, Fgf8 is also expressed in pharyngeal endoderm (e) and splanchnic as well as more dorsal, paraxial, mesoderm $(\mathrm{m})$ and in the primitive streak (ps). (L) In six-somite embryos, PAX2 expression in the ectoderm of the prospective placode (pp) colocalizes with ectodermal expression of the FGF8/GFP fusion protein in $K .(M, N)$ By eight somites, Fgf8 expression in the surface ectoderm is excluded from the dorsal otic placode (pp) but is maintained in the ventral ectoderm. Expression in the pharyngeal endoderm (e) is maintained. Expression is additionally detected in the telencephalon $(t)$. Expression is absent from hindbrain neural ecto$\operatorname{derm}(\mathrm{n})$.

splanchnic mesoderm, and underlying pharyngeal endoderm (Fig. 6F,G); this pattern was unchanged at the sixsomite stage (Fig. 6I,J). In addition to ectodermal and endodermal FGF8/GFP expression, even stronger and more dorsally situated expression occurred in the mesenchyme of embryos at the five- and six-somite stages (Fig. $6 \mathrm{H}, \mathrm{K}$ ). This localization of FGF8/GFP to the otic region was confirmed by colocalization of the ectodermal expression domain with PAX2 (Fig. 6K,L). By the eight-somite stage, Fgf8 transcripts still remained in surface ectoderm ventral to the placodal ectoderm, but were no longer found in the otic placode (Fig. 6M,N). In addition, Fgf8 expression was detected in the pharyngeal endoderm and in the intervening mesoderm (Fig. 6M,N). Thus, during the period of otic induction, Fgf 8 was expressed in or in close proximity to tissues required for otic induction, as well as briefly in the preplacodal ectoderm itself. Unlike in zebrafish embryos (Phillips et al. 2001; Leger and Brand 2002; Maves et al. 2002), mouse Fgf8 transcripts were never detected in the hindbrain.

Fgf8 is required redundantly with Fgf3 for mouse otic induction

Fgf8 null embryos die during gastrulation, precluding analysis of otic development (Sun et al. 1999). We asked whether Fgf8 plays a role in otic induction in the mouse by drastically reducing its expression in embryos using hypomorphic $\left(F g f 8^{H}\right)$ and null $\left(F g f 8^{-}\right)$alleles. $F g f 8^{H /-}$ pups survive until shortly after birth, when they die of heart defects (Moon and Capecchi 2000). Because the signals required for otic induction are redundant, we also removed $F g f 3$ to reveal a function for Fgf8. Therefore, $\mathrm{Fgf3}^{+/-} ; \mathrm{Fgfl}^{+/ H}$ and $\mathrm{Fgf3}^{+/-} ; \mathrm{Fgfl}^{+/-}$animals were intercrossed and offspring were collected between E8.0 and E9.5 and genotyped. Of 305 total offspring, 19 had the $\mathrm{Fgfl}^{-/-} ; \mathrm{Fgf \textrm {S } ^ { H / - }}$ genotype (referred to subsequently as Fgf3/Fgf8 "double mutants"), which is the expected number. This suggests that the levels of Fgf8, even in the absence of $F g f 3$, are sufficient to support development through the stages of interest.

Otic development was assessed at the vesicle stage using Pax2 as a marker. Control E9.5 $\mathrm{Fgf3}^{+/-} ; \mathrm{Fgfl}^{+/-} \mathrm{em}$ bryos formed normal otic vesicles expressing Pax2 in a medial domain (Fig. 7A,B). $\mathrm{Fgf3}^{+/+} ; \mathrm{Fg}_{\mathrm{fO}} \mathrm{HI}^{/-}$or $\mathrm{Fgf3}^{-/-} ; \mathrm{Fgfl}^{+/+}$ embryos also formed otic vesicles with appropriately localized Pax2 expression (Fig. 7C-F). In striking contrast, all five E9.5 Fgf3/Fgf8 double mutants failed to form otic vesicles (Fig. 7G,H), showing that $F g f 3$ and $F g f 8$ are required redundantly for mouse otic vesicle formation.

Next, we examined a panel of otic markers on control and $F g f 3 / F g f 8$ double-mutant embryos at E8.0, when the otic placode was forming. In control embryos, Pax2 was expressed as normal in a domain encompassing the earforming region that in cross-section marked the surface ectoderm (Fig. 7I,J); the dorsal region of this domain forms the otic placode. In double-mutant embryos, Pax2 was expressed in the ventral ectoderm, but not in the dorsal, ear-forming region of the ectoderm (Fig. 7K,L). Similarly, another early marker of the otic placode, Pax8, was expressed throughout the ectoderm of control embryos (Fig. $7 \mathrm{M}, \mathrm{N}$ ), but was excluded from the dorsal ectoderm of double-mutant embryos (Fig. 7O,P). The dorsal surface ectoderm of the double-mutant embryos was thin, characteristic of uninduced ectoderm (Fig. 7O,P). Similarly staged embryos with the $\mathrm{Fgf3}^{-/-} ; \mathrm{Fgfl}^{+/-}$or $\mathrm{Fgf3}^{+/-} ; \mathrm{Fgfl}^{\mathrm{H} /-}$ genotype had normal patterns of Pax2 and Pax8 expression (data not shown). In summary, our results show that the earliest stages of otic induction require both $F g f 3$ and $F g f 8$. 


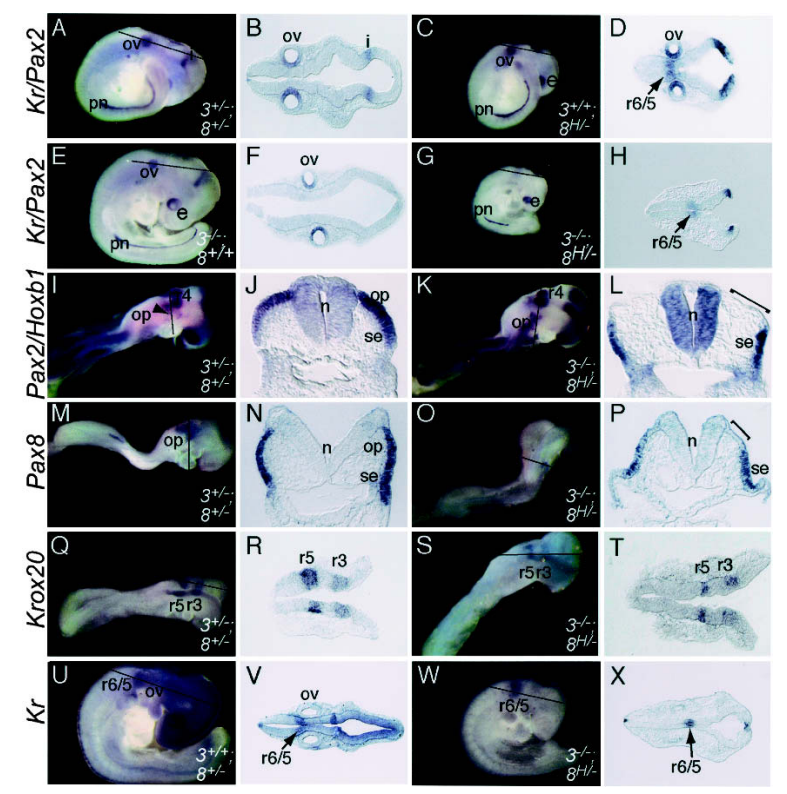

Figure 7. Otic, but not hindbrain, development is blocked in Fgf3/Fgf8 double-mutant embryos. $(A, C, E, G, I, K, M, O, Q, S, U, W)$ E8.5-9.5 control and mutant embryos were processed for in situ hybridization with the probes indicated to the left of each row. Embryos were sectioned in either the coronal $(B, D, F, H, R, T, V, X)$ or the transverse plane $(J, L, N, P)$. The section taken through the otic region (the plane is indicated by a line through each embryol is shown in the panel to the right of each whole embryo. Whole-mount images are shown in the lateral view with rostral to the right. The genotype of each embryo is indicated to the bottom right of each whole-mount panel. $(A-H)$ E9.5 Fgf3/Fgf8 double mutants, but not $\mathrm{Fgf3}^{-/-}$or $F g f 8^{\mathrm{H/-}}$ mutants, fail to develop otic vesicles (ov) or express Pax2 in the otic region. The $\mathrm{Kr}$ probe also included in this experiment was unsuccessful in labeling the control hindbrain $(A, B)$, so its absence in the Fgf3 mutant $(E, F)$ could not be interpreted. However, the $K r$ probe did label the Fgf8 mutant $(C, D)$ and double-mutant $(G, H)$ hindbrains, suggesting that $\mathrm{r} 5$ and $\mathrm{r} 6$ were normal in these embryos. $(I-L)$ In E8.5 control embryos, Pax2 is expressed throughout the otic placode (op) and ventral surface ectoderm (se). In Fgf3/Fgf8 double mutants, Pax2 expression is lost in the dorsal surface ectoderm (indicated by a bracket). Hoxb1 expression in $\mathrm{r} 4$ is not affected in Fgf3/Fgf8 double mutants. $(M-P)$ At E8.5, Pax8 expression occurs throughout the otic placode (op) and ventral surface ectoderm (se) of control embryos. In Fgf $3 / F g f 8$ double mutants, Pax 8 expression is lost in the dorsal surface ectoderm (indicated by a bracket). (Q-T) Krox 20 expression in $\mathrm{r} 3$ and $\mathrm{r} 5$ is not affected in $F g f 3 / F g f 8$ double mutants. $(U-X) K r$ expression in r6 and r5 is not affected in Fgf3/Fgf8 double mutants. (pn) Pronephros; (i) isthmus; (e) eye; (n) neural ectoderm.

\section{Hindbrain patterning is normal in Fgf3/Fgf8 double-mutant mouse embryos}

Simultaneous loss of Fgf3 from the hindbrain and Fgf10 from the mesoderm underlying the otic placode also blocks mouse otic induction and does so in the absence of major hindbrain patterning defects (Wright and Mansour 2003a). In contrast, simultaneous depletion of zebrafish Fgf3 and Fgf8, both of which are expressed in the hindbrain and are required redundantly for otic induc- tion, has major effects on hindbrain patterning (Maves et al. 2002; Walshe et al. 2002). To determine whether loss of mouse $\mathrm{Fgf3}$ and $\mathrm{Fgf} 8$ affects otic vesicle formation via effects on the hindbrain, we used a panel of hindbrain markers: Hoxb1 to mark r4 (Fig. 7I-L), Krox20 to mark r3 and r5 (Fig. 7Q-T), and $K r / M a f b$ to mark r5 and r6 (Fig. 7U-X). The labeling of double-mutant embryos could not be distinguished from that of control embryos, suggesting that hindbrain patterning defects are not responsible for the failure of otic induction in $\mathrm{Fgf} / \mathrm{Fg} f 8$ doublemutant embryos.

Progressive reduction of Fgf3 expression in the Fgf8 hypomorphic background has quantitative effects on mouse otic induction that are similar to those in the Fgf10 mutant background

$F g f 3$ and $F g f 10$ play quantitative and distinct roles in otic induction. Examination of embryos with both combinations of three mutant alleles using ventromedial (Pax2) and dorsal $(D 1 \times 5)$ markers showed that loss of Fgf3 affects otic gene expression and vesicle position and size more severely than does loss of Fgf10 (Wright and Mansour 2003a). To determine whether the same is true for Fgf3 and Fgf8, we hybridized similarly staged embryos (21-25 somites) of all relevant intermediate genotypes with $G b x 2$, a marker of the dorsomedial otic cup and vesicle, and compared these to each other as well as to a matched set of slightly younger (17-19 somites) Fgf3 and Fgf10 mutant embryos. As expected, just after otic vesicle closure, Gbx2 was detected in the dorsomedial region of control vesicles, which were of normal size and dorsal position (Fig. 8A,B). The normal dorsomedial localization of $G b x 2$ expression and dorsal position of the otic vesicle was seen in embryos with strongly reduced levels of Fgf8 and normal or 50\% reduced levels of Fgf3, although both the latter embryos and vesicles were small relative to the control (Fig. 8C,D,G,H). In contrast, otic expression of $G b x 2$ was diminished, but still appropriately localized, in embryos lacking Fgf3 only, and these vesicles had a slightly ventralized position (Fig. 8E,F). Gbx2 expression was entirely absent from the otic vesicles of embryos lacking Fgf3 and having only $50 \%$ of normal Fgf8 levels, and in these embryos the position of the vesicle was even more ventral and the size was clearly reduced (Fig. 8I,J). Finally, Fgf3/Fgf8 double mutants did not express $G b \times 2$ in the "otic" region because they failed to form otic vesicles (Fig. 8K,L). Thus, Fgf3 and $F g f 8$ have quantitative and distinct roles in otic vesicle formation.

Gbx2 expression and otic morphology were also examined at the late otic cup stage in a group of embryos having similar combinations of Fgf3 and Fgf10 null alleles. As expected, double heterozygotes showed robust expression of Gbx2 in the dorsomedial otic cup (Fig. $8 \mathrm{M}, \mathrm{N})$. The same results were obtained in embryos lacking Fgf10 and having normal (Fig. $8 \mathrm{O}, \mathrm{P}$ ) or $50 \%$ reduced levels of Fgf3 (Fig. 8S,T). The embryo lacking Fgf3 only had little expression of $G b x 2$ in one otic vesicle and normal expression in the other (Fig. 8Q,R). This variable 


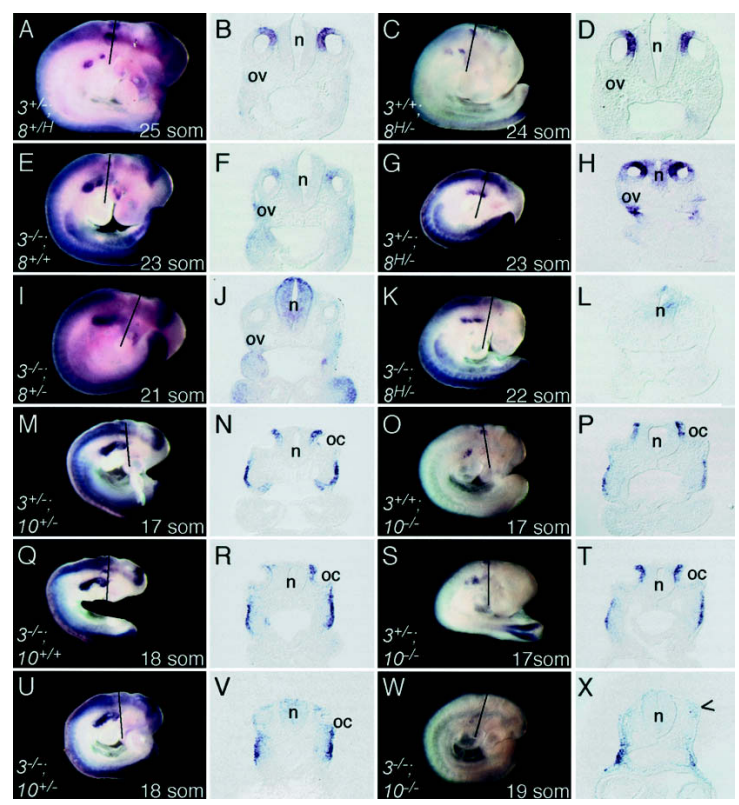

Figure 8. In the absence of $F g f 3$, otic development has a similar dependence on Fgf8 and Fgf10. E9.5 embryos isolated from intercrosses of the Fgf3 and Fgf8 alleles or the Fgf3 and Fgf10 alleles were processed for in situ hybridization with a $G b x 2$ probe and sectioned in the transverse plane. A section taken through the otic region (the plane is indicated by a line through each embryol is shown in the panel to the right of each whole embryo. Whole-mount images are labeled with the genotype and somite (som) number and shown in the lateral view with rostral to the right. $(A-L)$ In control, $\mathrm{Fgfl}^{+/+} ; \mathrm{Fgf}^{\mathrm{H} /-}$, and $\mathrm{Fgf3}^{+/-} ; \mathrm{Fgf}^{\mathrm{H/-}}$ embryos, Gbx2 is expressed normally in the dorsomedial aspect of the otic vesicle (ov). In $\mathrm{Fgf3}^{-/-} ; \mathrm{Fgfl}^{+/+}$and $\mathrm{Fgf3}^{-/-} ; \mathrm{Fgf}^{+/-}$embryos, Gbx2 expression is diminished and absent, respectively. In $F g f 3 / F g f 8$ double mutants, no otic vesicle and no $G b x 2$ expression is detected. $(M-X)$ In control, $\mathrm{Fgf3}^{+/+} ; \mathrm{FgflO}^{-/-}$, and $\mathrm{Fgf3}^{+/-} ; \mathrm{FgflO}^{-/-}$embryos $\mathrm{Gbx} 2$ is expressed normally in the dorsomedial region of the otic cup (oc). In the $\mathrm{Fgf3}^{-/-} ; \mathrm{Fg} f 1 \mathrm{O}^{+/+}$embryo the level of $\mathrm{Gbx} 2$ expression is diminished in one otic cup, illustrating the variable expressivity of this phenotype. In $\mathrm{Fgf3}^{-/} ; \mathrm{FgflO}^{+/-}$and $\mathrm{Fgf3} / \mathrm{FgflO}$ double-mutant embryos $G b x 2$ expression is absent from the otic region. In $\mathrm{Fgf3/Fgf8}$ double mutants $(K)$, this absence of $G b \times 2$ expression is coincident with the complete loss of recognizable otic tissue. However, some disorganised ectodermal tissue (devoid of $G b \times 2$ expression) is seen in the $\mathrm{Fgf3}^{-/-} ; \mathrm{Fg} f 1 \mathrm{O}^{-/-}$embryo (arrowhead). (n) Neural ectoderm.

expressivity of the Fgf3 allele has been shown previously (Mansour et al. 1993; Wright and Mansour 2003a). Notably, the embryo lacking Fgf3 and with 50\% reduced levels of Fgf10 (Fig. 8U,V) showed no otic expression of Gbx2 and had otic cups that were relatively small and ventrally and laterally displaced from the hindbrain. Finally, as expected, otic development was severely disrupted in the Fgf3/Fgf10 double-mutant embryo (Fig. $8 \mathrm{~W}, \mathrm{X})$. One side of the embryo showed no otic development and the other side had some organized epithelial tissue that was larger, but less well organized than the microvesicles previously found in $50 \%$ of $\mathrm{Fgf3} / \mathrm{Fgf10}$ double-mutant embryos (Wright and Mansour 2003a).
No otic expression of $G b x 2$ was detected in this embryo. Thus, with reduced or absent Fgf3, loss of Fgf8 or Fgf10 has similar consequences to otic development.

\section{Induction or maintenance of mouse mesenchymal Fgf10 expression depends on Fgf3 and Fgf8}

One explanation for the similarity between $\mathrm{Fgf3} / \mathrm{Fgf8}$ and Fgf3/Fgf10 intermediate and double-mutant phenotypes is that mouse $F g f 8$, like chick $F g f 8$, is required for normal expression of the mouse mesodermal otic inducer, FGF10. Thus, we evaluated Fgf10 expression in Fgf3/Fgf8 double-mutant embryos. At both preplacodal stages examined (zero and eight somites), there was a clear reduction of mesenchymal Fgf10 in double-mutant embryos relative to control embryos (Fig. 9, cf. A,C and B,D). Although Fgf10 expression was not completely lost, it was clear that the most dorsal regions of the mesenchyme, underlying the preplacodal ectoderm, was free of Fgf10 transcripts. In contrast, mesenchymal expression of $G b \times 2$ was not affected in double-mutant embryos (Fig. 9E,F), suggesting that Fgf3 and Fgf8 are required redundantly to induce or maintain normal levels of mesenchymal Fgf10 expression, but that mesenchymal gene expression is not globally dependent on these signals.

\section{Discussion}

Induction and early development of the inner ear are regulated by the expression of genes in the adjacent neural ectoderm and mesoderm. In chick and mouse, both inducing tissues provide FGF signals that are required for or are strongly implicated in otic induction. We show that in chick, a third tissue, the endoderm, is also re-

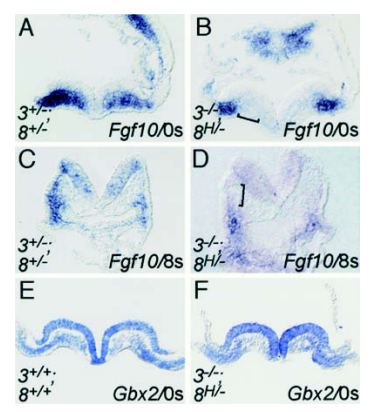

Figure 9. The expression of mesodermal $F g f 10$ is reduced in Fgf3/Fgf8 double mutants. Preplacodal control $(A, C, E)$ and $F g f 3 /$ $F g f 8$ double-mutant $(B, D, F)$ mouse embryos were hybridized with Fgf10 or Gbx2 as indicated in each panel and sectioned in the transverse plane. $(A, B)$ Sections taken through the otic region of zero somite embryos show a reduction in Fgf10 expression in the medial mesoderm (indicated with a bracket). $(C, D)$ Sections taken through the otic region of eight-somite embryos show a reduction in Fgf10 expression in the mesenchyme underlying the dorsal ectoderm, where the otic placode is expected to form. $(E, F)$ Sections taken through the otic region of zerosomite embryos show that mesodermal Gbx2 expression is unaffected in Fgf3/Fgf8 double-mutant embryos. 
quired for otic induction. This tissue expresses Fgf8, and our experiments provide evidence that FGF8 is both sufficient and necessary for normal expression of the gene encoding the mesodermal otic inducer, FGF19, thereby initiating otic development. In mouse, Fgf8 is expressed by the endoderm, as well as by other otic-inducing regions, and it is required, in addition to Fgf3, for expression of the gene encoding the mesodermal otic inducer, FGF10, and for subsequent otic induction. Thus, in both species, a cascade of FGF signals drives otic development.

The tissue sources of FGF signals potentially relevant for initiating otic induction

It is now well established that the hindbrain and underlying mesoderm are major sources of otic-inducing signals. Tissue ablation and transplantation studies, as well as observations of classic and targeted mutants, show that these tissues are necessary and sufficient for induction of otic placode gene expression and for induction of placodal morphology and subsequent development (for reviews, see Baker and Bronner-Fraser 2001; Kiernan et al. 2002; Riley and Phillips 2003). FGF signals are clearly required for the earliest stages of otic induction as well as for subsequent development of the otic placode and vesicle (for reviews, see Riley and Phillips 2003; Wright and Mansour 2003b; Mansour and Schoenwolf 2005). The four major vertebrate models (Xenopus, zebrafish, chick, and mouse) all express Fgf3 in dorsal neural ectoderm adjacent to preplacodal tissue (Wilkinson et al. 1988; Tannahill et al. 1992; Mahmood et al. 1995; Raible and Brand 2001), and at least in zebrafish and mice, an FGF3 signal is required (redundantly with another FGF signal) for induction of the otic placode. In zebrafish, Fgf3 is partially redundant for otic placode induction with Fgf8, which is coexpressed in the hindbrain (Phillips et al. 2001; Leger and Brand 2002; Maroon et al. 2002). In mouse, Fgf3 is required redundantly for otic placode induction with Fgf10, which is expressed weakly and mainly ventrally in neural ectoderm, and is more strongly expressed in mesenchyme underlying the developing neural ectoderm and preplacodal ectoderm (Alvarez et al. 2003; Wright and Mansour 2003a). Fgf3 is also expressed in the chick hindbrain and can induce some aspects of otic development (Mahmood et al. 1995; Vendrell et al. 2000), but its requirement for otic induction in this species remains to be tested. The role of Fgf3 in Xenopus ear development has not been addressed.

The mesoderm underlying the preplacodal ectoderm is also a source of FGF signals, at least in chick and mouse. Mesodermal FGF19, in synergy with a WNT8c signal, induces chick otic gene expression (Ladher et al. 2000). Genetic and expression analyses suggest that mesenchymal Fgf10 may assume this role in mouse (Wright and Mansour 2003a). Zebrafish mutants deficient in mesoderm have abnormal otic phenotypes (Mendonsa and Riley 1999; Leger and Brand 2002). As the mutated genes are required to specify mesendoderm, any structure dependent on mesodermal signals would be affected indi- rectly. Obvious candidates for direct otic-inducing signals include zebrafish FGF19 and FGF10 (Ng et al. 2002; Katoh 2003). Another possibility is FGF8, which in addition to its role as a neural ectodermal otic inducer, is expressed in cranial paraxial mesoderm (Reifers et al. 2000; Thisse et al. 2001).

We have identified a third tissue in chick otic placode induction. Endoderm ablation routinely reduced the size of the otic placode. The failure to completely eliminate otic induction suggests either that other tissues supply redundant otic-inducing signals and/or regeneration restores the required signal. Jacobson (1963) similarly concluded that the endoderm was required for otic induction when he found that explanted preplacodal ectoderm was most effectively induced as ear when endoderm was included with mesoderm and neural ectoderm. Other examples of inductive signaling by endoderm include development of the pharyngeal cartilage (Couly et al. 2002; David et al. 2002; Ruhin et al. 2003) and epibranchial placodes (Begbie et al. 1999). However, it is unlikely that endoderm plays a direct role in otic induction; rather, it does so by inducing mesodermal otic-inducing signals.

\section{FGF8 as an initiator of otic development acting to induce mesodermal FGFs}

Several lines of evidence implicate FGF8 as an inducer of mesodermal FGFs functioning in otic placode induction. Prior to otic induction, chick Fgf8 is expressed in the same endodermal tissue required for otic induction, but not in other otic-inducing tissues. Furthermore, knockdown of chick Fgf8 reduced expression of mesodermal Fgf19 and inhibited otic placode formation. Rescue of Pax2 expression by FGF19 shows not only that Fgf8 acts indirectly in otic induction, but also that FGF19 is required.

In mouse, the expression pattern of $\mathrm{Fgf8}$ is more complex than in chick. Fgf8 is detected in the periotic region in endoderm, mesenchyme, and preplacodal ectoderm during the four- to six-somite stages, just prior to the appearance of the otic placode. However, mesenchymal expression of Fgf8 actually begins much earlier, in the cardiac crescent (splanchnic mesoderm), whose caudal extremes lie in close proximity to the developing otic regions. Our genetic data reveal that mouse Fgf8 is required redundantly with $F g f 3$ for otic induction. The similarity of the Fgf3/Fgf8 and Fgf3/Fgf10 double-mutant otic phenotypes and intermediate three mutant allele phenotypes, coupled with the reduction of Fgf10 in the Fgf3/Fgf8 double-mutant embryos, argues that mouse FGF8 functions to induce or maintain mesenchymal Fgf10 expression, much as chick FGF8 induces mesodermal Fgf19 expression. FGF8 may mediate this effect at least in part through one of its preferred receptors, FGFR3c, which is expressed in the mesenchyme /data not shown). FGF8 is unlikely to signal directly to the preplacodal ectoderm, as this tissue does not express appropriate receptors (Ornitz et al. 1996; Wright and Man- 
sour 2003a). Experiments to determine the tissue source of Fgf8 required for mouse otic induction are underway.

\section{Common features of FGF signaling in otic induction among species and similarities to the roles of FGF signaling in limb development}

We propose that there are both serial and parallel FGF signals required for otic induction and that a generally similar scheme applies to all vertebrate species. The first FGF signal acts on the mesoderm to induce a second FGF signal. In chick, the first signal (FGF8) arises from the endoderm, whereas in mouse, the FGF8 signal could arise from any of the three periotic germ layers. Functional evidence for the initiating FGF signal remains to be obtained in other species. The second FGF signal, FGF19 in chick and FGF10 in mouse, acts in parallel with a third signal from the hindbrain. In chick, WNT8c, expressed by the hindbrain, acts synergistically with FGF19 to induce the otic placode, suggesting that WNT8c is a hindbrain signal for otic induction. However, Fgf3 is also expressed in the chick hindbrain, where it could also function as a hindbrain signal. In mouse, FGF3 and FGF10 are required redundantly for otic placode induction, and FGF3 likely functions as a hindbrain signal since global (Mansour et al. 1993) or hindbrainspecific FGF3 reduction (McKay et al. 1996) have similar effects on otic vesicle development. In addition, the Hoxa1 null otic abnormalities can be rescued with retinoic acid, which induces hindbrain, but not otic, Fgf3 expression (Pasqualetti et al. 2001). In zebrafish, as discussed earlier, the identity of the mesodermal signal is unknown, but $F g f 3$ and $F g f 8$ encode redundant hindbrain signals for otic induction and hindbrain patterning. Together, the hindbrain and mesodermal FGFs induce the placodal genes required for this tissue to take on an otic fate.

Serial and parallel FGF signaling also characterize limb development. Studies of chick and mouse embryos show that FGF8 and possibly another FGF from the intermediate mesoderm induce FGF10 expression in the lateral plate mesoderm, which grows out during limb bud formation. Mesodermal FGF10 is in turn required to induce FGF8 in the limb apical ectodermal ridge (AER), and these two factors are subsequently required to maintain each other's expression while the limb bud grows and is patterned (for reviews, see Martin 1998; Capdevila and Izpisua Belmonte 2001). Furthermore, some of AER FGF8 function is redundant with FGF4 (Sun et al. 2002; Boulet et al. 2004). A generally similar scheme also acts during zebrafish fin development. Recently, a new mesenchymal Fgf gene, Fgf24, has been placed upstream of Fgf10 during the very earliest stages of fin bud initiation (Fischer et al. 2003). It will be interesting to explore in greater detail other potential parallels between ear and limb development, including whether otic induction, like limb induction, involves positive and negative feedback loops and the same downstream targets, applying what we learn from one system to understand the other.

\section{Materials and methods}

\author{
Endoderm extirpations
}

Extirpations of regions of chick embryos at stages 4 and 5 (Hamburger and Hamilton 1951) were performed in Early Chick (EC) culture. Using an eyebrow knife, tissue was removed and drawn away from the embryo by aspiration ablating the endoderm fated to underlie the otic-inducing mesoderm. Embryos were then placed into a humidified incubator at $37^{\circ} \mathrm{C}$ for either $8 \mathrm{~h}$ until stage 7 or 8 to assess changes to $\mathrm{Fg} f 19$ expression or for $36 \mathrm{~h}$ to monitor otic induction using Pax2 as a marker for the inner ear primordium.

\section{Tissue recombinations}

Preparations of endoderm or mesoderm were isolated from explants using $5 \mathrm{U} / \mathrm{mL}$ of dispase for 2 min followed by manual dissection in saline containing $2 \%$ fetal bovine serum. These were recombined in a collagen drop (Ladher et al. 2000) and cultured for $8 \mathrm{~h}$. Rostral endoderm from stage 4 quail embryos was defined as that endoderm anterior to Hensen's node. Caudal endoderm was defined as endoderm posterior to Hensen's node. Chick mesoderm was explanted at stage 5 .

\section{Protein treatment}

Chick stage 5 rostral mesoderm was isolated from explants using dispase treatment as described above. FGF4 and FGF8 (both obtained from R\&D Systems) were applied individually to heparin-coated acrylic beads at a concentration of $500 \mu \mathrm{g} / \mathrm{mL}$. Explants treated with protein-coated beads were grown in Neurobasal media with $\mathrm{B} 27$ supplement at $37^{\circ} \mathrm{C}$ in $5 \% \mathrm{CO}_{2}$.

\section{Electroporation}

A pSilencer 1.0 vector (Ambion) containing an Fgf8 cDNA construct was introduced into chick embryos. The construct containing a DNA duplex formed from two oligonucleotides $\left(5^{\prime}\right.$ GCACGTGCAGATCTTGGACTTCAAGAGAAGTCCAAGA TCTGCACGTGCTTTTTT-3' and 5'-AATTAAAAAAGCACG TGCAGATCTTGGACTCTCTTGAAGTCCAAGATCTGCA CGTGCGGCC-3'), when transcribed from the U6 promoter, formed an $F g f 8$ hairpin RNA. The Fgf8-interfering construct and a Venus-tagged Histone for use as a tracer (Okita et al. 2004) were injected subendodermally into stage 4 chick embryos. Five $7-\mathrm{V}$ pulses of $50 \mathrm{msec}$ each, with 100 -msec intervals, were applied using a CUY-21 electroporator (Nepagene) through flat paddle-shaped electrodes. The embryos were cultured for an additional 4-36 h.

Rescue of $F g f 8$ siRNA effects was tested by using three-layered tissue explants (Ladher et al. 2000; explant " $\mathrm{a}$ ") of electroporated embryos; unelectroporated explants served as positive controls. Electroporated explants were treated with FGF19 beads as described previously (Ladher et al. 2000).

\section{Whole-mount in situ hybridization}

Chick and mouse embryos were stained in whole mount with digoxigenin-labeled RNA probes and 14- $\mu \mathrm{m}$ frozen sections were prepared as described previously (Ladher et al. 2000; Wright and Mansour 2003a). Chick Fgf8 expression was detected using a probe described previously (Crossley et al. 1996). A species-specific probe derived from the 3' UTR was used to detect expression of chick Fgf19 (Ladher et al. 2000). This probe did not cross-react with quail mRNA (data not shown). Chick otic development was assessed using Pax2 (Ladher et al. 2000). 
Mouse Fgf8 expression was determined by staining wild-type CD-1 embryos staged according to the day following detection of a vaginal plug and/or by counting somites with an Fgf8 exon 5 probe (Moon and Capecchi 2000). Use and origins of the other mouse in situ probes were as described previously (Wright and Mansour 2003a).

Fluorescent immunohistochemistry

for detection of FGF8 expression

Fgf $8^{\mathrm{GFP} / \mathrm{GFP}}$ homozygous males (Macatee et al. 2003) were mated to HPRT-Cre females (Tang et al. 2002) to generate embryos bearing a recombined $F g f 8^{\mathrm{GFPR}}$ allele. These embryos produced a hybrid FGF8-GFP fusion protein in all Fgf8 mRNA-ex-

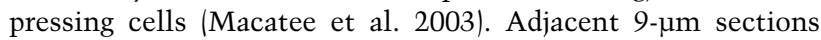
were analyzed for GFP or PAX2 protein localization. GFP was detected with rabbit anti-GFP primary antiserum in combination with a FITC-conjugated secondary antibody $(1: 1000$ and 1:500, respectively, both from Molecular Probes). PAX2 was detected in adjacent sections by using a rabbit polyclonal primary antiserum (Covance, 1:50) and Texas Red-conjugated secondary antibody (Molecular Probes, 1:500). Immunohistochemical detection of the fusion protein was vastly more sensitive than was in situ hybridization to Fgf8 mRNA.

\section{Generation of Fgf3/Fgf8-deficient mice}

The targeted alleles of Fgf3 (Fgf3-, presumptive null) and Fgf8 $\left(F g f 8^{H}\right.$, hypomorphic and $F g f 8^{-}$, null) alleles were described previously (Mansour et al. 1993; Moon and Capecchi 2000). $\mathrm{Fgf3}^{+-} ; \mathrm{Fgfl}^{+/-}$and $\mathrm{Fgf3}^{+/-} ; \mathrm{Fgfl}^{+/ H}$ parents were intercrossed to generate experimental embryos. Genotypes for Fgf3 and Fgf8 alleles were determined using PCR amplification of yolk sac or tail DNA as described previously (Moon and Capecchi 2000; Wright and Mansour 2003a). All crosses and genotyping of Fgf3 and Fgf10 mutant alleles were performed as described previously (Wright and Mansour 2003a).

\section{Acknowledgments}

We thank Dr. Timm Schroeder for the Histone-H2B/Venus fusion construct. Albert Noyes, Jaclyn Tygesen, and Tim Macatee provided excellent technical assistance. This work was funded by the NIDCD (DC00473: T.J.W.; DC04185: G.C.S., S.L.M.), the Ministry of Science, Education and Culture of Japan, and the MEXT Leading Projects (R.K.L.).

\section{References}

Adamska, M., Herbrand, H., Adamski, M., Kruger, M., Braun, T., and Bober, E. 2001. FGFs control the patterning of the inner ear but are not able to induce the full ear program. Mech. Dev. 109: 303-313.

Alvarez, I.S. and Navascues, J. 1990. Shaping, invagination, and closure of the chick embryo otic vesicle: Scanning electron microscopic and quantitative study. Anat. Rec. 228: 315326.

Alvarez, Y., Alonso, M.T., Vendrell, V., Zelarayan, L.C., Chamero, P., Theil, T., Bosl, M.R., Kato, S., Maconochie, M., Riethmacher, D., et al. 2003. Requirements for FGF3 and FGF10 during inner ear formation. Development 130: 63296338.

Anniko, M. and Wikstrom, S.O. 1984. Pattern formation of the otic placode and morphogenesis of the otocyst. Am. J. Otolaryngol. 5: 373-381.
Baker, C.V. and Bronner-Fraser, M. 2001. Vertebrate cranial placodes I. Embryonic induction. Dev. Biol. 232: 1-61.

Begbie, J., Brunet, J.F., Rubenstein, J.L., and Graham, A. 1999. Induction of the epibranchial placodes. Development 126: 895-902.

Boulet, A.M., Moon, A.M., Arenkiel, B.R., and Capecchi, M.R. 2004. The roles of Fgf4 and Fgf8 in limb bud initiation and outgrowth. Dev. Biol. 273: 361-372.

Capdevila, J. and Izpisua Belmonte, J.C. 2001. Patterning mechanisms controlling vertebrate limb development. Annu. Rev. Cell Dev. Biol. 17: 87-132.

Couly, G., Creuzet, S., Bennaceur, S., Vincent, C., and Le Douarin, N.M. 2002. Interactions between Hox-negative cephalic neural crest cells and the foregut endoderm in patterning the facial skeleton in the vertebrate head. Development 129: 1061-1073.

Crossley, P.H., Minowada, G., MacArthur, C.A., and Martin, G.R. 1996. Roles for FGF8 in the induction, initiation, and maintenance of chick limb development. Cell 84: 127136.

David, N.B., Saint-Etienne, L., Tsang, M., Schilling, T.F., and Rosa, F.M. 2002. Requirement for endoderm and FGF3 in ventral head skeleton formation. Development 129: 44574468.

Fischer, S., Draper, B.W., and Neumann, C.J. 2003. The zebrafish fgf24 mutant identifies an additional level of Fgf signaling involved in vertebrate forelimb initiation. Development 130: 3515-3524.

Groves, A.K. and Bronner-Fraser, M. 2000. Competence, specification and commitment in otic placode induction. Development 127: 3489-3499.

Hamburger, V. and Hamilton, H.L. 1951. A series of normal stages in the development of the chick embryo. J. Morphol. 88: 49-92.

Jacobson, A.G. 1963. The determination and positioning of the nose, lens and ear. I. Interactions within the ectoderm, and between the ectoderm and underlying tissues. J. Exp. Zool. 154: 273-283.

- 1966. Inductive processes in embryonic development. Science 152: 25-34.

Karabagli, H., Karabagli, P., Ladher, R.K., and Schoenwolf, G.C. 2002. Comparison of the expression patterns of several fibroblast growth factors during chick gastrulation and neurulation. Anat. Embryol. (Berl.) 205: 365-370.

Katoh, M. 2003. Evolutionary conservation of CCND1ORAOV1-FGF19-FGF4 locus from zebrafish to human. Int. J. Mol. Med. 12: 45-50.

Kiernan, A.E., Steel, K.P., and Fekete, D.M. 2002. Development of the mouse inner ear. In Mouse development: Patterning morphogenesis and organogenesis (eds. J. Rossant and P. Tam), pp. 539-566. Academic Press, San Diego.

Ladher, R.K., Anakwe, K.U., Gurney, A.L., Schoenwolf, G.C., and Francis-West, P.H. 2000. Identification of synergistic signals initiating inner ear development. Science 290: 19651967.

Lawson, A. and Schoenwolf, G.C. 2003. Epiblast and primitivestreak origins of the endoderm in the gastrulating chick embryo. Development 130: 3491-3501.

Leger, S. and Brand, M. 2002. Fgf8 and Fgf3 are required for zebrafish ear placode induction, maintenance and inner ear patterning. Mech. Dev. 119: 91-108.

Macatee, T.L., Hammond, B.P., Arenkiel, B.R., Francis, L., Frank, D.U., and Moon, A.M. 2003. Ablation of specific expression domains reveals discrete functions of ectoderm- and endoderm-derived FGF8 during cardiovascular and pharyngeal development. Development 130: 6361-6374. 
Mahmood, R., Kiefer, P., Guthrie, S., Dickson, C., and Mason, I. 1995. Multiple roles for FGF-3 during cranial neural development in the chicken. Development 121: 1399-1410.

Mansour, S.L. and Schoenwolf, G.C. 2005. Morphogenesis of the inner ear. In Springer handbook of auditory research (eds. D.K. Wu and M.W. Kelley). Springer-Verlag (in press).

Mansour, S.L., Goddard, J.M., and Capecchi, M.R. 1993. Mice homozygous for a targeted disruption of the proto-oncogene int-2 have developmental defects in the tail and inner ear. Development 117: 13-28.

Maroon, H., Walshe, J., Mahmood, R., Kiefer, P., Dickson, C., and Mason, I. 2002. Fgf3 and Fgf8 are required together for formation of the otic placode and vesicle. Development 129: 2099-2108.

Martin, G.R. 1998. The roles of FGFs in the early development of vertebrate limbs. Genes \& Dev. 12: 1571-1586.

Maves, L., Jackman, W., and Kimmel, C.B. 2002. FGF3 and FGF8 mediate a rhombomere 4 signaling activity in the zebrafish hindbrain. Development 129: 3825-3837.

McKay, I.J., Lewis, J., and Lumsden, A. 1996. The role of FGF-3 in early inner ear development: An analysis in normal and kreisler mutant mice. Dev. Biol. 174: 370-378.

Mendonsa, E.S. and Riley, B.B. 1999. Genetic analysis of tissue interactions required for otic placode induction in the zebrafish. Dev. Biol. 206: 100-112.

Moon, A.M. and Capecchi, M.R. 2000. Fgf8 is required for outgrowth and patterning of the limbs. Nat. Genet. 26: 455-459.

Ng, J.K., Kawakami, Y., Buscher, D., Raya, A., Itoh, T., Koth, C.M., Rodriguez Esteban, C., Rodriguez-Leon, J., Garrity, D.M., Fishman, M.C., et al. 2002. The limb identity gene Tbx 5 promotes limb initiation by interacting with Wnt $2 b$ and Fgf10. Development 129: 5161-5170.

Okita, C., Sato, M., and Schroeder, T. 2004. Generation of optimized yellow and red fluorescent proteins with distinct subcellular localization. Biotechniques 36: 418-422, 424.

Ornitz, D.M., Xu, J., Colvin, J.S., McEwen, D.G., MacArthur, C.A., Coulier, F., Gao, G., and Goldfarb, M. 1996. Receptor specificity of the fibroblast growth factor family. J. Biol. Chem. 271: 15292-15297.

Pasqualetti, M., Neun, R., Davenne, M., and Rijli, F.M. 2001. Retinoic acid rescues inner ear defects in Hoxal deficient mice. Nat. Genet. 29: 34-39.

Phillips, B.T., Bolding, K., and Riley, B.B. 2001. Zebrafish fgf3 and fgf 8 encode redundant functions required for otic placode induction. Dev. Biol. 235: 351-365.

Raible, F. and Brand, M. 2001. Tight transcriptional control of the ETS domain factors Erm and Pea3 by Fgf signaling during early zebrafish development. Mech. Dev. 107: 105-117.

Reifers, F., Bohli, H., Walsh, E.C., Crossley, P.H., Stainier, D.Y., and Brand, M. 1998. Fgf8 is mutated in zebrafish acerebellar (ace) mutants and is required for maintenance of midbrainhindbrain boundary development and somitogenesis. Development 125: 2381-2395.

Reifers, F., Walsh, E.C., Leger, S., Stainier, D.Y., and Brand, M. 2000. Induction and differentiation of the zebrafish heart requires fibroblast growth factor 8 (fgf8/acerebellar). Development 127: 225-235.

Represa, J., Leon, Y., Miner, C., and Giraldez, F. 1991. The int-2 proto-oncogene is responsible for induction of the inner ear. Nature 353: 561-563.

Riley, B.B. and Phillips, B.T. 2003. Ringing in the new ear: Resolution of cell interactions in otic development. Dev. Biol. 261: 289-312.

Ruhin, B., Creuzet, S., Vincent, C., Benouaiche, L., Le Douarin, N.M., and Couly, G. 2003. Patterning of the hyoid cartilage depends upon signals arising from the ventral foregut endo- derm. Dev. Dyn. 228: 239-246.

Sun, X., Meyers, E.N., Lewandoski, M., and Martin, G.R. 1999. Targeted disruption of Fgf8 causes failure of cell migration in the gastrulating mouse embryo. Genes \& Dev. 13: 18341846.

Sun, X., Mariani, F.V., and Martin, G.R. 2002. Functions of FGF signalling from the apical ectodermal ridge in limb development. Nature 418: 501-508.

Tang, S.H., Silva, F.J., Tsark, W.M., and Mann, J.R. 2002. A Cre/loxP-deleter transgenic line in mouse strain 129S1/ SvImJ. Genesis 32: 199-202.

Tannahill, D., Isaacs, H.V., Close, M.J., Peters, G., and Slack, J.M. 1992. Developmental expression of the Xenopus int-2 (FGF-3) gene: Activation by mesodermal and neural induction. Development 115: 695-702.

Thisse, B., Pflumio, S., Furthauer, M., Loppin, B., Heyer, V., Degrave, A., Woehl, R., Lux, A., Steffan, T., Charbonnier, X., et al. 2001. Expression of the zebrafish genome during embryogenesis. ZFIN [http://zfin.org/cgi-bin/webdriver?MIval= aa-pubview2.apg\&OID=ZDB-PUB-010810-1].

Vendrell, V., Carnicero, E., Giraldez, F., Alonso, M.T., and Schimmang, T. 2000. Induction of inner ear fate by FGF3. Development 127: 2011-2019.

Walshe, J., Maroon, H., McGonnell, I.M., Dickson, C., and Mason, I. 2002. Establishment of hindbrain segmental identity requires signaling by FGF3 and FGF8. Curr. Biol. 12: 11171123.

Wilkinson, D.G., Peters, G., Dickson, C., and McMahon, A.P. 1988. Expression of the FGF-related proto-oncogene int-2 during gastrulation and neurulation in the mouse. EMBO $\mathrm{I}$. 7: 691-695.

Wright, T.J. and Mansour, S.L. 2003a. Fgf3 and Fgf10 are required for mouse otic placode induction. Development 130: 3379-3390.

. 2003b. FGF signaling in ear development and innervation. Curr. Top. Dev. Biol. 57: 225-259.

Wright, T.J., Ladher, R.K., McWhirter, J., Schoenwolf, G.C., and Mansour, S.L. 2004. Mouse FGF15 is the ortholog of chick FGF19, but is not uniquely required for otic induction. Dev. Biol. 269: 264-275. 


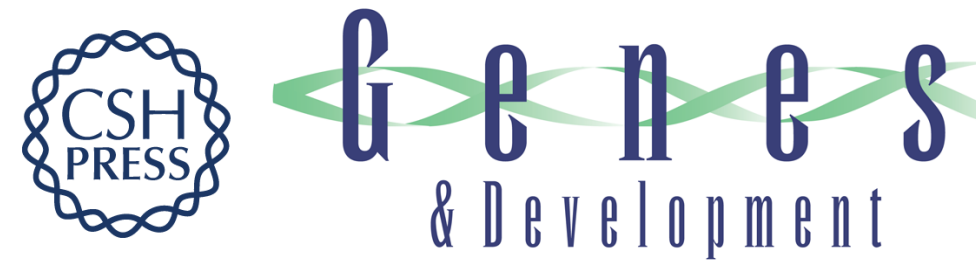

\section{FGF8 initiates inner ear induction in chick and mouse}

Raj K. Ladher, Tracy J. Wright, Anne M. Moon, et al.

Genes Dev. 2005, 19:

Access the most recent version at doi:10.1101/gad.1273605

References This article cites 48 articles, 20 of which can be accessed free at: http://genesdev.cshlp.org/content/19/5/603.full.html\#ref-list-1

License

Email Alerting Receive free email alerts when new articles cite this article - sign up in the box at the top Service right corner of the article or click here.

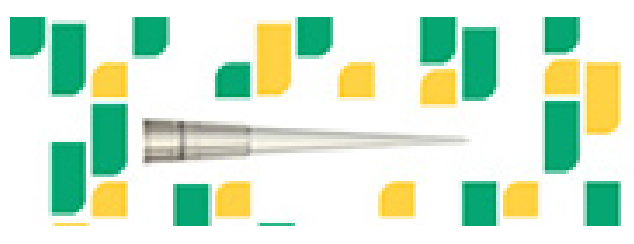

Focused on your science. 